\title{
Compatibility of a diffractive pupil and coronagraphic imaging
}

\author{
Eduardo Bendek ${ }^{1,2}$, Ruslan Belikov ${ }^{2}$, Eugene Pluzhnik ${ }^{2}$ and Olivier Guyon ${ }^{1,3}$ \\ 1: University of Arizona, 2: NASA Ames Research Center, 3: Subaru Telescope.
}

\begin{abstract}
Detection and characterization of exo-earths require direct-imaging techniques that can deliver contrast ratios of $10^{10}$ at 100 milliarcseconds or smaller angular separation. At the same time, astrometric data is required to measure planet masses and can help detect planets and constrain their orbital parameters. To minimize costs, a single space mission can be designed using a high efficiency coronograph to perform direct imaging and a diffractive pupil to calibrate wide-field distortions to enable high precision astrometric measurements. This paper reports the testing of a diffractive pupil on the high-contrast test bed at the NASA Ames Research Center to assess the compatibility of using a diffractive pupil with coronographic imaging systems. No diffractive contamination was found within our detectability limit of $2 \times 10^{-7}$ contrast outside a region of $12 \lambda / \mathrm{D}$ and $2.5 \times 10^{-6}$ within a region spanning from 2 to $12 \lambda / \mathrm{D}$. Morphology of the image features suggests that no contamination exists even beyond the detectability limit specified or at smaller working angles. In the case that diffractive contamination is found beyond these stated levels, active wavefront control would be able to mitigate its intensity to $10^{-7}$ or better contrast.
\end{abstract}

Keywords: Extrasolar Planets, Astronomical Instrumentation. 


\section{INTRODUCTION}

The direct detection of planetary systems such as Beta Pictoris, HR8799, Fomalhaut, and more recently the (indirect) detection of a large number of new exoplanet candidates by the Kepler mission have motivated not only scientists but also the general public to wonder about other habitable worlds and consider them as a real possibility.

Traditional detection techniques such as radial velocity and transits have allowed us to detect exoplanets with a wide range of distance and size. However, characterization of planets that resemble the Earth and can host life will require spectroscopy to determine their atmospheric composition, and spectroscopy requires direct imaging, at least for nontransiting or small planets (Levine et al., 2009). High precision astrometry is also necessary to determine the mass and orbit of the planet much more precisely than direct imaging can by itself. Direct imaging requires large contrast ratios of $10^{10}$ with respect to the host star, and very small angular separation on the order of 100 milliarcseconds and smaller; a challenging requirement that necessitates a very aggressive coronagraph.

Researchers have proposed a large number of coronagraph variations to achieve high contrast imaging; however, there are only a few that are able to theoretically achieve the required contrast at $2 \lambda / \mathrm{D}$. In particular, the Phase-Induced Amplitude Apodization Coronograph (PIAA) (Belikov et al., 2010; Guyon, 2003) offers very high contrast using lossless apodization achieved by highly aspheric optics. Given the importance of direct imaging for determination of exoplanet habitability and the aggressive performance of PIAA, it has been proposed for several space missions such as PECO (Guyon et al., 2010a), EXCEDE (Greene et al., 2007), and in general scales to many different telescope sizes. Furthermore, it is already in operation on the sky with an extreme AO system on 
the Subaru telescope (Guyon et al., 2010b).

Despite the potential of direct imaging in spectroscopic characterization, the precise mass and orbit properties of the planet enabled by high precision astrometry are necessary to properly evaluate the habitability of a planet (Lunine et al., 2008; Shao et al., 2010). High precision astrometry is one of the most promising techniques to determine the mass and orbit of exoplanets because it solves the inclination ambiguity present in planets detected with the radial velocity (RV) method, it is more sensitive to longerperiod planets than $\mathrm{RV}$ where the habitable zone is expected, and it is about 10 times less sensitive to the effects of sun spots than RV approaches (Seager, 2010). Star spots and stellar variability in general set the ultimate limit for indirect planet detection.

When astrometric measurements of a star are obtained, differences in field distortion of the imaging system between different epochs sets the largest term of the astrometry error budget. To solve this problem a diffractive distortion mapping system has been proposed (Guyon et al., 2012a) that allows calibrating wide field distortions using diffractive spikes. A laboratory to demonstrate this novel technique is being developed at The University of Arizona (Bendek et al., 2011). The use of a diffractive grid for narrow-field astrometry applications has also been proposed (Marois et al., 2006; Sivaramakrishnan \& Oppenheimer 2006).

The combination of direct imaging and high precision astrometry is essential to assess the habitability of planets in the habitable-zone, as it provides estimates of the planet mass, orbit, effective temperature, and atmosphere/surface composition. It is therefore essential to advance both techniques and demonstrate that both measurements can be performed in a single mission using a monolithic mirror telescope in space, instead of 
two separate missions, as was originally thought necessary. This combination would dramatically reduce the cost and schedule required to obtain both measurements and would reliably assess the habitability of exoplanets (Guyon et al., 2012b).

This paper focuses on measuring the effect of the diffractive pupil over the coronographic Field of View (FoV). The main goal is to demonstrate that the diffractive spikes behave as predicted by the models and do not introduce any contamination of the Inner Working Angle (IWA) that could diminish the chronographic capability of a combined mission, and therefore showing the compatibility of both techniques on a single mission.

\section{METHODOLOGY AND EXPERIMENTAL SETUP}

2.1. The diffractive pupil mirror and its design parameters

For this experiment a one-inch diameter flat folding mirror acts as pupil and stop for the system in the astrometry test bed and is imprinted with dots on its coating. The design of the dot pattern in the pupil plane defines the geometry of the diffraction spikes in the image plane as predicted by the Fraunhofer diffraction expression which relates the electric field at pupil, $U_{S}^{+}\left(x_{s}, y_{s}\right)$ and the resultant field at the image plane $U_{o}\left(x_{o}, y_{o}\right)$ using a Fourier transform, as described by equation 1 .

$$
U_{o}\left(x_{o}, y_{o}\right)=-\frac{j e^{j k z_{o}}}{\lambda z_{o}} \exp \left[\frac{j k}{2 z_{o}}\left(x_{o}^{2}+y_{o}^{2}\right)\right] F_{\eta=\frac{y_{o}}{\lambda z_{o}}} F_{\xi=\frac{x_{o}}{\lambda z_{o}}}\left[U_{S}^{+}\left(x_{S}, y_{S}\right)\right]
$$

From the nature of the Fourier Transform, it is inferred that the radius of the spike envelope is inversely proportional to the dot size and the period of spikes is inversely proportional to the period of the dots. The number of spikes is set by the periodicity of the spike pattern. Considering these two parameters and the wavelength range of the 
source, the appropriate dot array and size can be selected to cover the FoV of the system with an adequate number diffraction spikes that provide a good distortion sampling. Figure 1 presents a simulation of the diffractive dots created on the image plane by monochromatic illumination of a pupil of diameter $D$ that has a hexagonal grid of dots, with a side of length $a$, and no dot in the center of each hexagon. For this particular design, a reciprocal hexagonal array of spots is created on the image plane with an angular separation between the first diffraction order and the optical axis in $\lambda / \mathrm{D}$ units given by,

$$
x_{1}=\frac{2}{3} \frac{D}{a} .
$$

Then a second order array of spots imaged in a hexagonal configuration is four times brighter and is rotated by 30 degrees. The angular separation, in $\lambda / D$ units, of those spots to the optical axis is given by,

$$
x_{2}=\frac{2 \sqrt{ } 3}{3} \frac{D}{a} .
$$

Higher order spots repeat at integer multiple distances of the first two orders. The brightness of the spots should also maintain a one to four ratio; however, the finite size of each spot induces an Airy function modulation that diminish the spot intensity as a function of distance to the optical axis and inversely proportional to the pupil dot size. 

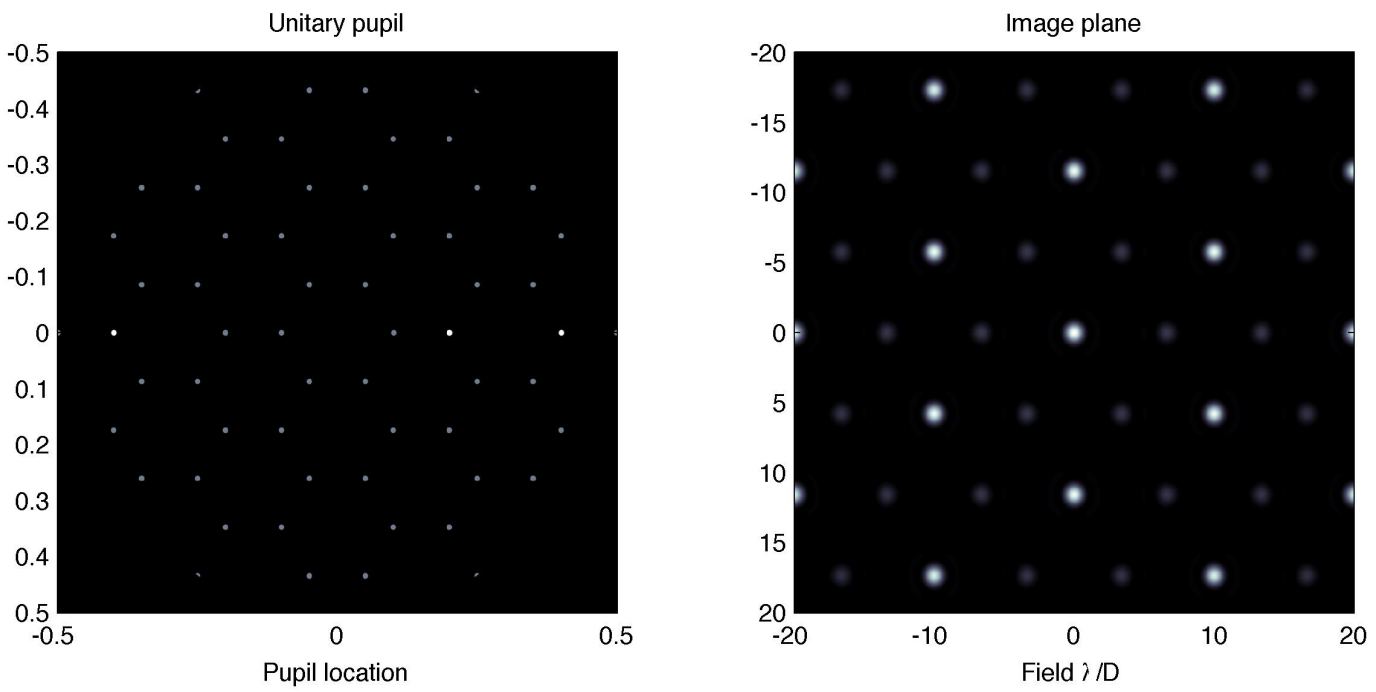

Figure 1. The diffractive grid pattern used for this experiment at the pupil is shown on the left and the resulting image is shown on the right. Second order spots are four times brighter than the first order ones close to the optical axis. Note that the pupil is unitary and the scale in the image plane is in $\lambda / \mathrm{D}$ units.

\subsection{The diffractive pupil mirror manufacturing}

The diffractive mirror used for this experiment was manufactured by the Colorado Nanofabrication Lab (CNL) using a chrome-on-glass composition that is exposed with a direct laser-writing tool. A thin layer of resist is coated on the chrome and the laser exposes the pattern of holes in the positive resist, which is washed away in a developing solution. The substrate is then placed in an etch solution; therefore, when the resist is washed away, the chrome is etched. The result after this process is a pattern of etched holes in an otherwise solid chrome film. The master pattern is then used in a modified mask aligner, where the master mask is brought in contact with a resist-coated aluminum 
mirror. The pattern is transferred onto the mirror's resist by exposing the combination with a UV lamp. Then, the developing and etching process is repeated to obtain holes in the aluminum mirror surface. For the mask being used at the laboratory, the dot pattern selected is hexagonal with a $50 \mu \mathrm{m}$ side and $5 \mu \mathrm{m}$ holes, representing $2.4 \%$ of the total area of the mirror. An image of the pupil mask mounted on the support is shown in Figure 2, and a microscope image of the surface is shown on the right.
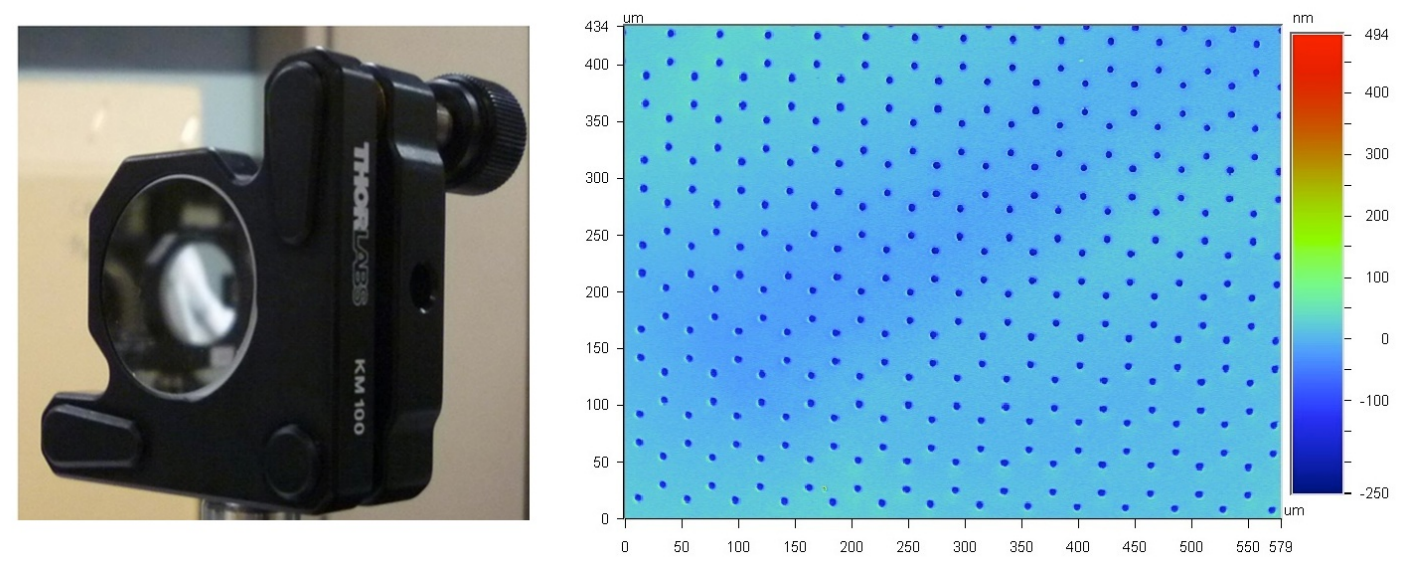

Figure 2. The image on the left shows a picture of the diffractive dotted mirror.

The image on the right shows a VEECO microscope image of the pupil mirror coating where the hexagonal dotted pattern be clearly seen. The dots are $5 \mu \mathrm{m}$ in diameter and hexagons are each $50 \mu \mathrm{m}$ wide. The coating is aluminum with a thickness of approximately $100 \mathrm{~nm}$.

\subsection{Experiment goal and setup}

The goal of this experiment is to demonstrate that the Diffractive Pupil (DP) concept is compatible with high contrast imaging techniques in the same telescope. This requires the diffractive orders on the image plane to be located outside the FoV of the coronograph, 
which by design should be attainable. However, manufacturing problems, such as low frequency variations on the spacing of the diffractive pupil dots, could generate unexpected diffraction orders inside the FoV of the coronagraph.

For this experiment we used the high contrast test bed at NASA Ames (Belikov et al., 2010). This test bed was designed to work down to $10^{-9}$ contrast levels using a PIAA coronograph, a Deformable Mirror (DM) to correct the wavefront, and a Focal Plane Occulter to mask the source. The bench was in air and thermally stabilized to $+/-0.002$ $\mathrm{K}$. The system has demonstrated contrast levels of $2 \times 10^{-8}$ raw contrast, which means that that no software post processing has been performed besides background subtraction, in an annular region that spans from 2.0 to $3.4 \lambda / \mathrm{D}$.

The diffractive pupil needed to be installed in or close to a pupil plane. Given the optical layout of the bench, the only available pupil plane was downstream from the PIAA coronograph where the DM was located. The DM was removed and a translation stage was installed. On the stage a flat mirror, with surface quality of $\lambda / 10$ at $633 \mathrm{~nm}$, and the diffractive pupil dotted mirror were installed and aligned. To select the diffractive pupil or the flat mirror, the stage was translated. After the pupil, a focal plane occulter was installed to block the source, avoiding saturation and bleeding of the camera. The optical layout is shown in Figure 3. 


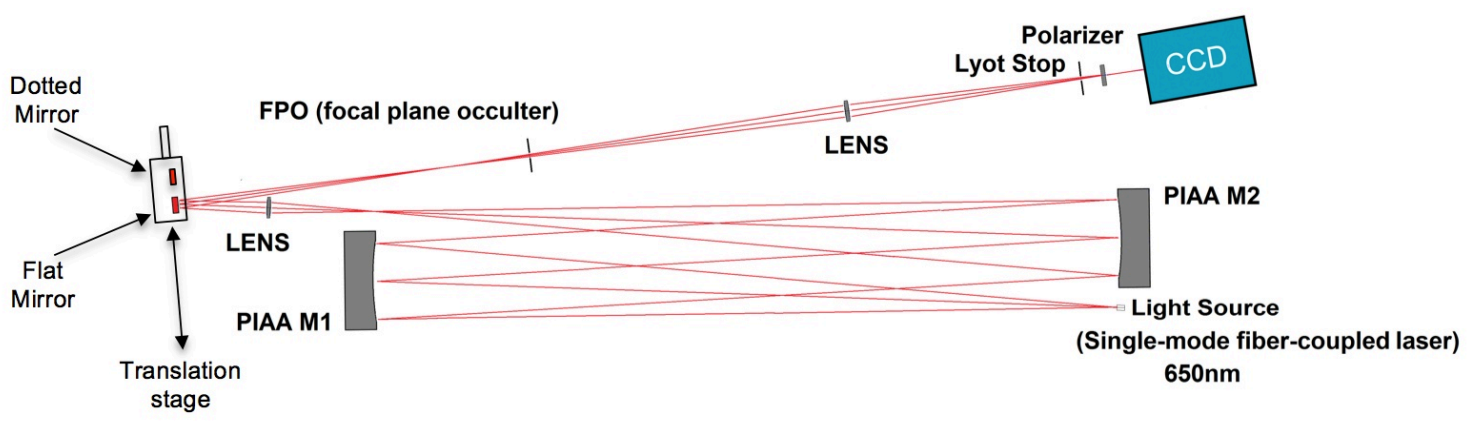

Figure 3. Optical layout of the high contrast imaging testbed. For this experiment the deformable mirror, which is in a pupil plane shown on the left side of the layout, was replaced by a diffractive pupil and a flat mirror that can be switched using a translation stage where the mirrors are mounted.

\subsection{Expected diffraction spot locations}

As it was explained in the previous section the diffractive pupil was installed after the PIAA system. Since this system concentrates light at the center of the pupil to achieve the apodization, it also modifies the plate scale of the system, so that a $1 \lambda / \mathrm{D}$ angle after the PIAA unit corresponds to an angle more than three times smaller on the sky (Guyon et al., 2005). In the real mission configuration, an inverse PIAA system would recover the normal plate scale of the system and correct for off-axis aberrations. However, the test bed did not have such a system available. As a result, two plate scales could be defined on the same image depending if we are analyzing a phenomena before or after PIAA. For this particular setup, the wide field images appeared to be 3.8 times closer to the optical axis than where they should have been if the astrometry mask was on the primary of the telescope and a reverse PIAA system was used. This magnification factor has to be applied to the camera plate scale, or "System units", to recover "on sky" units. 
A simulation of the diffractive pupil used for this experiment was performed to compare the predicted position and intensity of the spots with the real location observed when the experiment was carried out. In this simulation, a linear cut of a monochromatic electric field at the diffractive pupil, shown in the upper left corner of Figure 4, was propagated to the image plane using equation 1 . This figure only shows a section of the pupil cut enlarged enough to see the details and spacing of the pupil dots, but the computation was performed for the full length of the pupil used. Our pupil diameter $D$ was set during the experiment to be $6.5 \mathrm{~mm}$ and the hexagon side of the grid was $50 \mu \mathrm{m}$. Using equation 2, we predicted an angular distance of the first diffraction order of 86.7 $\lambda / \mathrm{D}$ in system units, and on the image it iswas found at $87.3 \lambda / \mathrm{D}$ system units, which is equivalent to $23 \lambda / \mathrm{D}$ "on sky" units. This corresponds to $0.7 \%$ difference between the predicted and the measured position of the diffractive spot, showing a good agreement with the model.

The second order spot can be seen on the upper right side of the image shown in Figure 4. This spot is found at $152 \lambda / \mathrm{D}$ system units, and equation 2 predicts its location at $150 \lambda / \mathrm{D}$ system units. This corresponds to less than $1 \%$ error between the model and the observations. The second order spot flux is measured to be 3.3 times the flux of the first order spots closer to the optical axis, which is dimmer to the 4 to 1 ratio due to the Airy function intensity modulation caused by the pupil dot size. Since the focus of this paper is in the IWA of the optical system and not on the location of the spots, from now on all angular units will be "on sky" because they are the units of interest for the IWA measurements. 

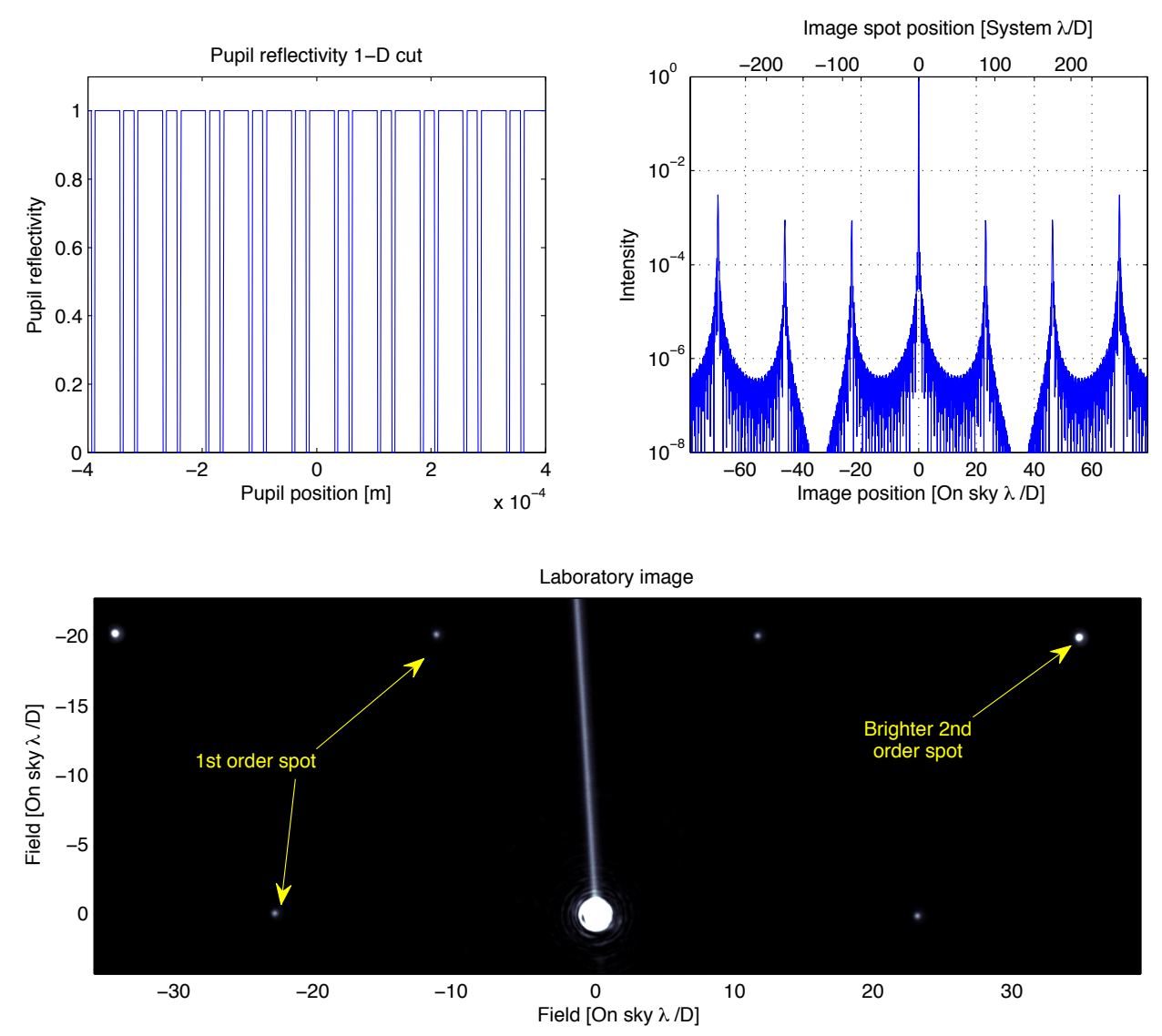

Figure 4. A linear cut of the electric field at the diffractive pupil is shown in the upper left corner and the image plane cut counterpart is shown on the right where the intensity and location of the future spots is predicted. The image on bottom shows the location and brightness the real spots observed of the focal plane.

In the case of the real mission, the beam will not be apodized before the diffractive pupil; hence the diffractive spots will have their own Airy rings that extend into the field of interest of the coronagraph. The diffractive spots would separate by about $100 \lambda / \mathrm{D}$ for the space mission design, and the total flux per diffraction spot (or spike in polychromatic light) would be about $10^{-5}$ of the central peak. At $100 \lambda / \mathrm{D}$, the Airy pattern is at $8 \times 10^{-8}$ 
contrast level, and the contrast contribution of the nearest diffraction spot/spike into the region of interest of the coronagraph is therefore $8 \times 10^{-8}$ times $10^{-5}$ that is in the order of $10^{-12}$ contrast. Even after accounting for the contribution of multiple spikes, this effect is therefore well below $10^{-10}$ required for direct exoplanet imaging and will not affect the coronagraphic performance of the mission.

\subsection{Experimental procedure and data reduction}

Due to the finite dynamic range of the camera, it was impossible to capture the full contrast level on a single exposure. Therefore, a set of images with different laser power settings was taken in order to calibrate the dynamic range of the images as the laser intensity increased. During the experiment sets of images were taken using the flat mirror and then using the diffractive dotted mirror. The resulting images were subtracted to detect any light contamination created by the diffractive mirror.

To maximize the contrast obtained by subtracting the flat and the diffractive mirror images, it was necessary to optimize two aspects of the image. The first one was to equalize the intensity of the images for the same laser power, which is different due to higher reflectivity of the flat mirror as well as natural laser power variations. The image created using the diffractive dotted mirror has about $20 \%$ less intensity than the flat mirror image. To compensate for this effect, a scaling factor that maximizes the contrast in the region of interest was computed and applied to the image. 

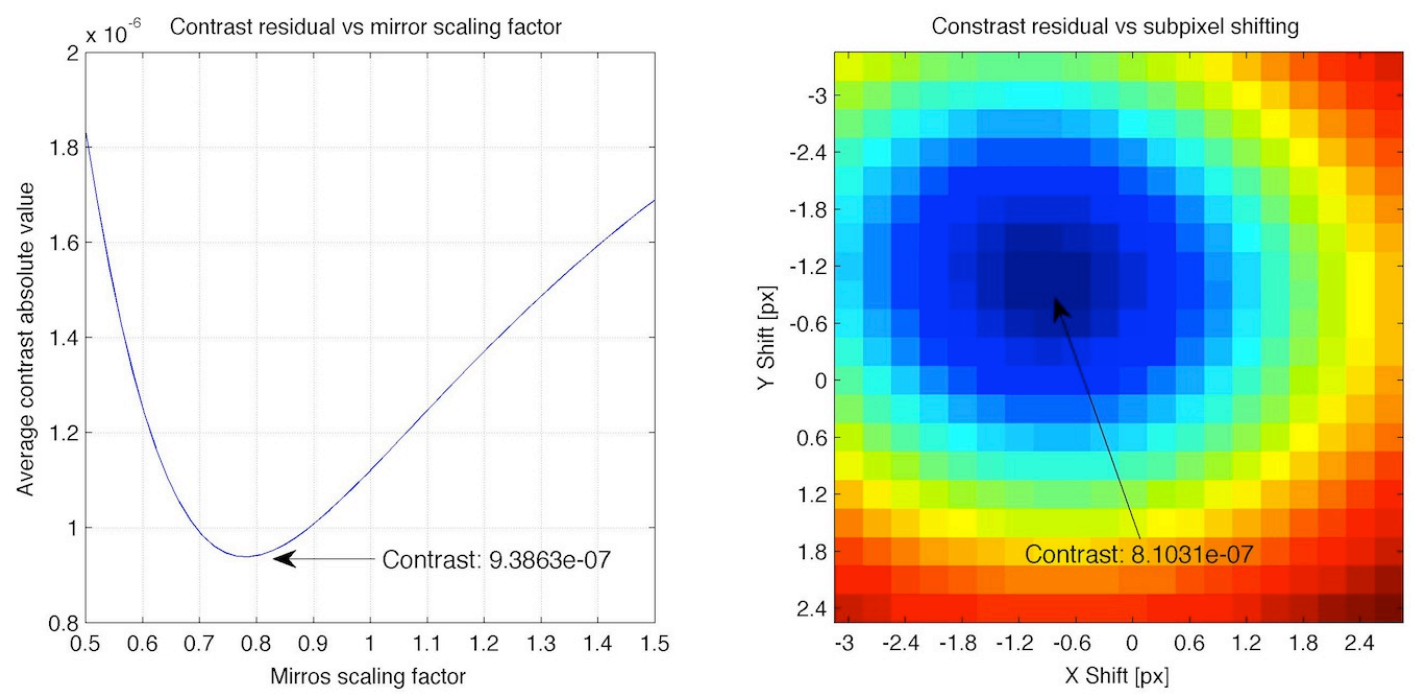

Figure 5. Intensity (left) and sub-pixel shift (right) contrast optimization plots. The brightness of the flat mirror image was reduced by factor of 0.8 to match the lower reflectivity of the diffractive pupil. A shift of $-0.9 \mathrm{px}$ and $-1.2 \mathrm{px}$ in $\mathrm{X}$ and $\mathrm{Y}$ axis is also applied to optimize the diffraction rings subtraction.

The second aspect is the image registration. The images needed to be displaced, on the order of 1 to 2 pixels, to overcome the relative alignment errors of the diffractive and flat mirror. The images were co-aligned using a contrast maximization merit function, which performs sub-pixel shifts and evaluation of the contrasts. After the correct shifts were calculated, the images were shifted to achieve optimal registration.

Figure 5 shows the average contrast obtained within a region of interest that spans from $2.1 \lambda / \mathrm{D}$ to $15 \lambda / \mathrm{D}$ versus the scaling factor value. An optimal contrast of $9.8 \times 10^{-7}$ is obtained when an image intensity factor of 0.78 is applied to the flat mirror image. After this correction was applied, the sub-pixel shifting was computed reaching an optimal contrast of $8.1 \times 10^{-7}$. The resulting image is shown in Figure 6. 


\section{RESULTS}

3.1 Contrast measurements using the occulter mask

For this measurement, 1s exposure images were taken with a laser current setting of $35 \mathrm{~mA}$. In this configuration the equivalent peak intensity of the central star behind the occulter was $1.44 \times 10^{9}$ counts. Three images using the flat mirror and the diffractive pupil were taken and averaged to reduce the noise. The resulting images were optimized and subtracted according to the procedure previously described. The resulting image is shown in Figure 6. The central source was blocked by the occulter, which spanned from 0 to $1.2 \lambda / \mathrm{D}$. Beyond this radius the image has ring-like features. The geometry of these rings is extremely sensitive to alignment of the system and therefore can change between exposures. As a result, the subtraction of the images does not cancel the rings completely. The diffraction orders are imaged as a grid of spots with inverse spatial frequency of the pupil grid as expected. They can be seen at approximately $23 \lambda / \mathrm{D}$ from the source. No spikes are created due to the narrow band illumination of the laser source. 


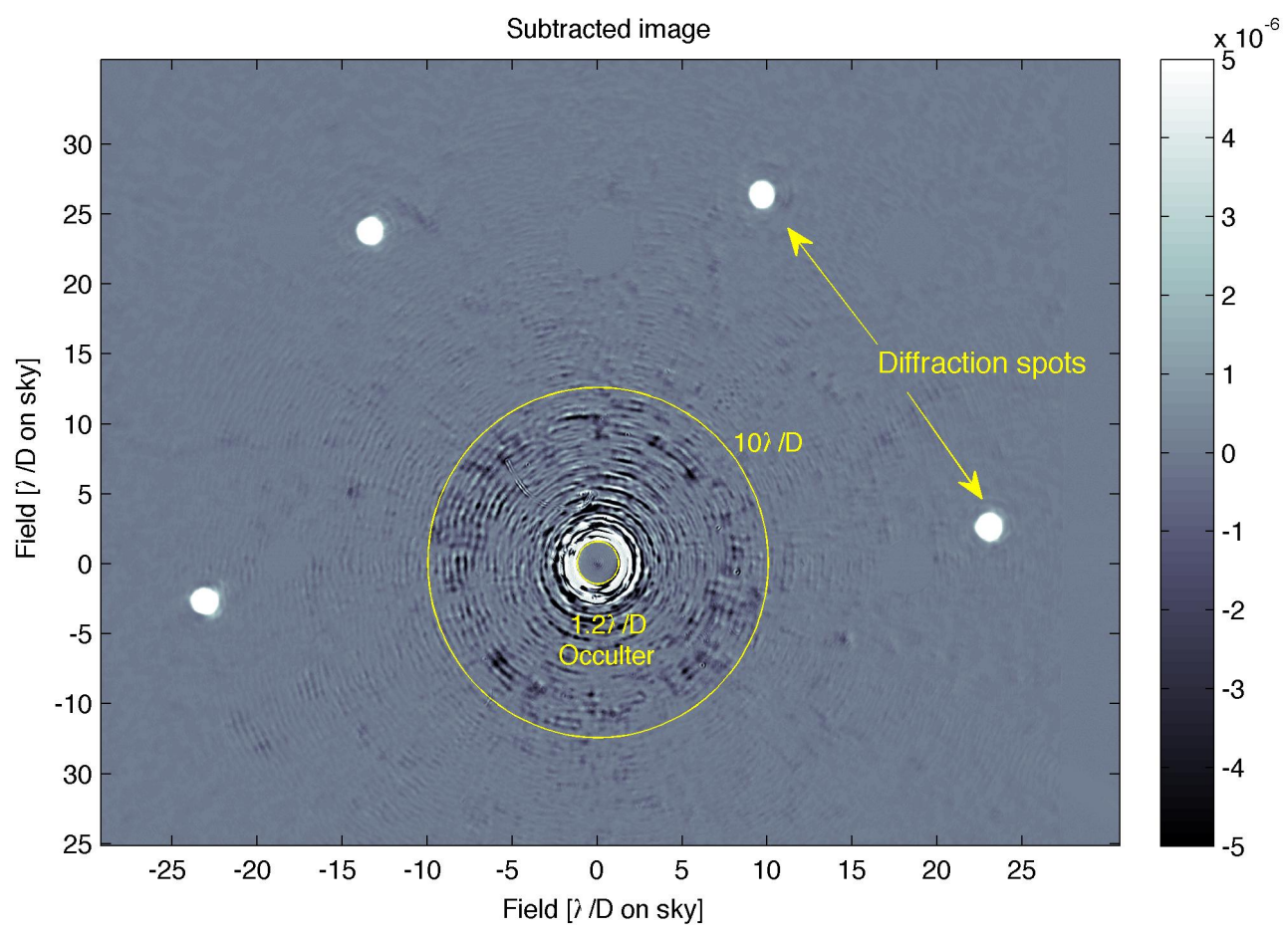

Figure 6. Differential image using the flat mirror and the diffractive dotted mirror. The occulter blocks the source with a radius of $1.2 \lambda / \mathrm{D}$. A circle of radius $10 \lambda / \mathrm{D}$ is shown for scale and comparison purposes with the PIAA focal plane wavefront errors shown in Figure 7 . The diffraction pattern created by the diffractive pupil in monochromatic laser light is imaged at approximately $23 \lambda / \mathrm{D}$ from the central star.

The resulting image after optimization has an average contrast of $8.1 \times 10^{-7}$ and there are no visible features created by the diffractive pupil at this level. Furthermore, we observe that coherent amplification enhances the intensity of the rings in some places. This kind of amplification is caused by the cross term created when the image intensity of the superposition of the electric field of different sources is computed as the square of the sum of their respective fields. 
The high contrast imaging test bed at NASA AMES uses two PIAA aspheric off-axis mirrors, which have minimal wavefront errors, causing phase errors on the pupil electric field resulting in speckles in different areas of the image. When the test bed is in normal operation, a deformable mirror is located where the diffractive pupil was installed. The DM performs active wavefront control to remove this and other sources of wavefront error. Figure 7, shows a map of this error induced by the PIAA mirrors.

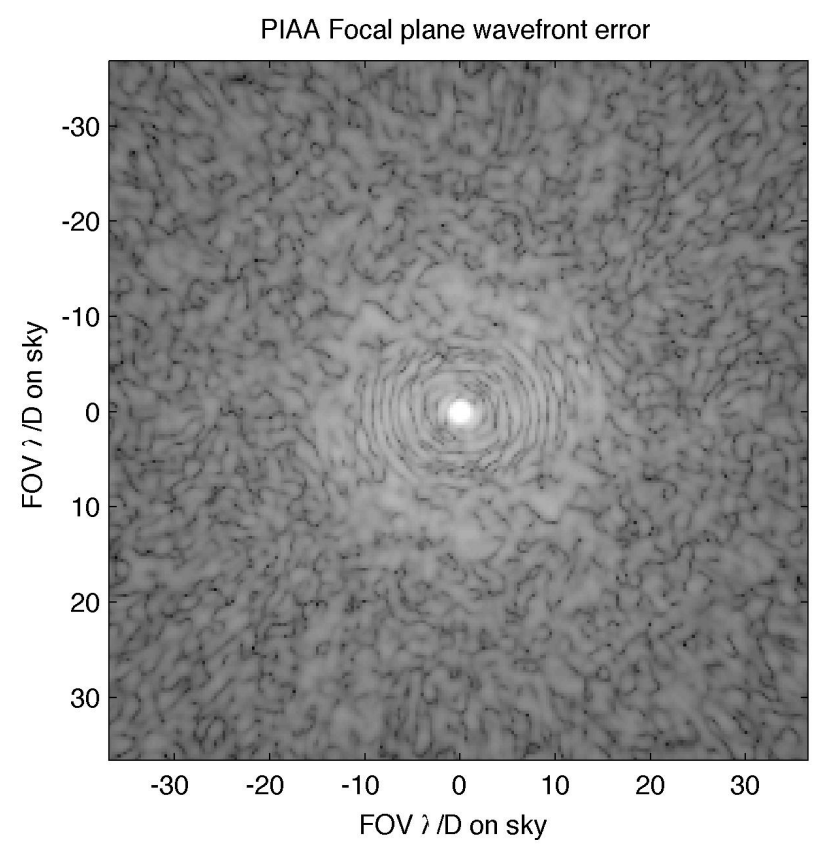

Figure 7. Simulation of the focal plane wavefront errors created by the PIAA mirrors. This perturbation on the electric field is probably coherently amplified when they overlap with the rings observed on the image.

To demonstrate that the diffraction rings' brightness enhancement is not caused by the diffractive mirror, we have compared the features and contrast level obtained when the diffractive and flat mirror images were subtracted, shown in the left column of Figure 8, and the subtraction of two subsequent images taken using the flat mirror without 
modifying any variables on the bench, which is shown in the column on the right. Note that in the former case the dynamic range has been adjusted to one order of magnitude tighter, to $+/-8 \times 10^{-7}$ for the image and to $+/-5 \times 10^{-7}$ for the plots showing the cuts.

From this experiment we can conclude the following: First, the diffraction rings cannot be completely subtracted because their shape changes from exposure to exposure even if the same flat mirror is used, and the bench is neither opened nor modified, as is shown in the second column of Figure 8. Second, the rings' intensity enhancement is probably caused by coherent amplification of the PIAA mirrors wavefront errors and not the diffractive mirror. By adjusting the dynamic range of the images and cuts of both columns in Figure 8, we show that the morphology of the features in the subtracted image is very similar when the diffractive mirror is used (left) and when is not (right). This fact strongly suggests that the diffractive mirror is not causing the features observed.
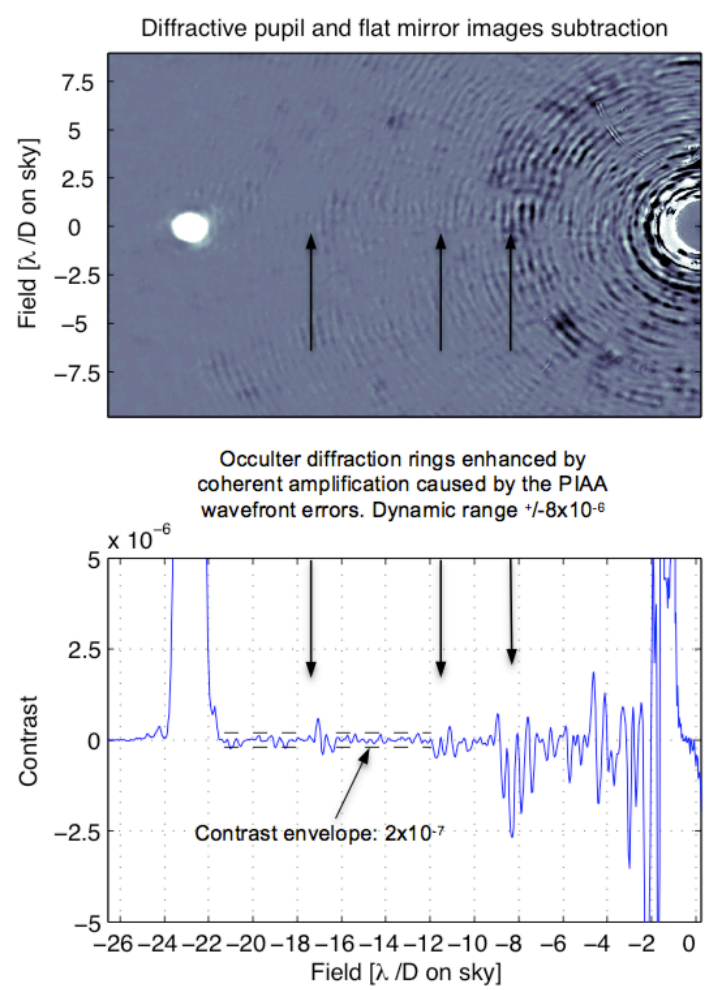

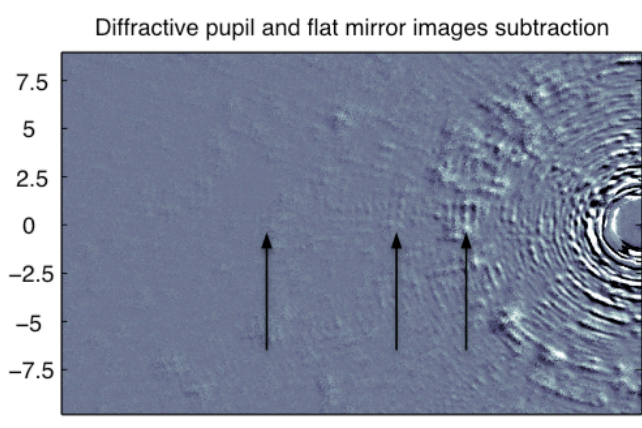

Occulter diffraction rings remain even using only flat mirror images showing that they are not a feature of the diffractive

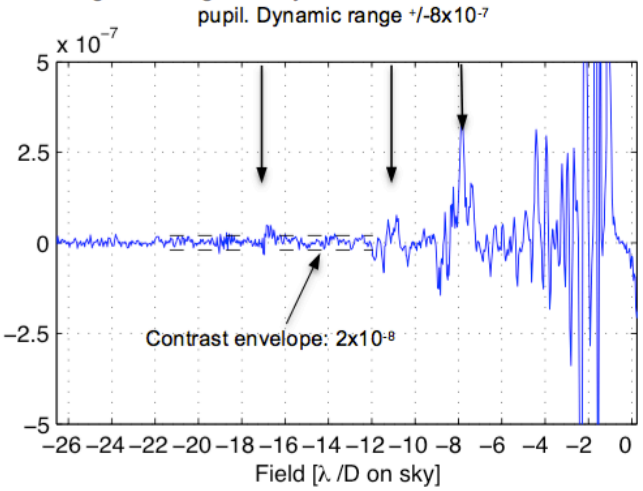


Figure 8 . The column on the left shows the subtraction and cut of the diffractive and flat mirror images. The column on the right shows the same for two subsequent images both taken using the flat mirror. The dynamic range is 10 times smaller in this case. Our contrast envelope or detectability limit is ${ }^{+} /-2 \times 10^{-7}$ for working angles larger than $12 \lambda / \mathrm{D}$ where coherent amplification is weaker.

Despite the fact that coherent amplification of the diffraction rings limits our ability to find contamination caused by the diffractive pupil, we still can distinguish different contrast envelopes. By inspecting several cuts on the image, we show that there is no contamination caused by the diffractive pupil for the following detectability limits; a contrast envelope of $2 \times 10^{-7}$ over a region outside $12 \lambda / \mathrm{D}, 5 \times 10^{-7}$ inside the region spanning from 10 to $12 \lambda / \mathrm{D}$, and $2.5 \times 10^{-6}$ between 10 to $2 \lambda / \mathrm{D}$. No adequate data subtraction was possible between 2 and $1.2 \lambda / \mathrm{D}$ where the edge of the occulter is located.

\section{DISCUSSION AND CONCLUSION}

We conclude that there is no diffractive contamination down to a contrast level of $2 \times 10^{-7}$ over the region outside $12 \lambda / \mathrm{D}$. If the morphology of the features are excluded from the contrast envelope, this result can be extended inside up to $5 \lambda / \mathrm{D}$. Inside the region spanning from 10 to $2 \lambda / \mathrm{D}$, the contrast is limited to $2.5 \times 10^{-6}$ ring patterns, which are probably created by the occulter and coherently amplified. Also, we note that in the region spanning from 5 to $10 \lambda / \mathrm{D}$, the contrast envelope gets tighter, down to a contrast level of $1 \times 10^{-6}$. Table 1 summarizes the detectability limits for different field location. 
The region spanning from 2 and $5 \lambda / \mathrm{D}$ is dominated by ringing features. As their intensity is maximally close to the occulter, a small misregstration of the images or shape change creates large contrast artifacts on the subtracted image. We ran the image registration optimization routine again setting the region of interest from 2 and $5 \lambda / \mathrm{D}$. After performing this optimization, we found no diffractive contamination up to a contrast envelope of $2.5 \times 10^{-6}$ within this region. Moreover, we consider it highly unlikely that diffractive features can appear in the region spanning from 2 and $5 \lambda / \mathrm{D}$ if no features have been detected at larger angles because it would require a single and very low spatial frequency component on the array of dots imprinted on the diffractive mirror.

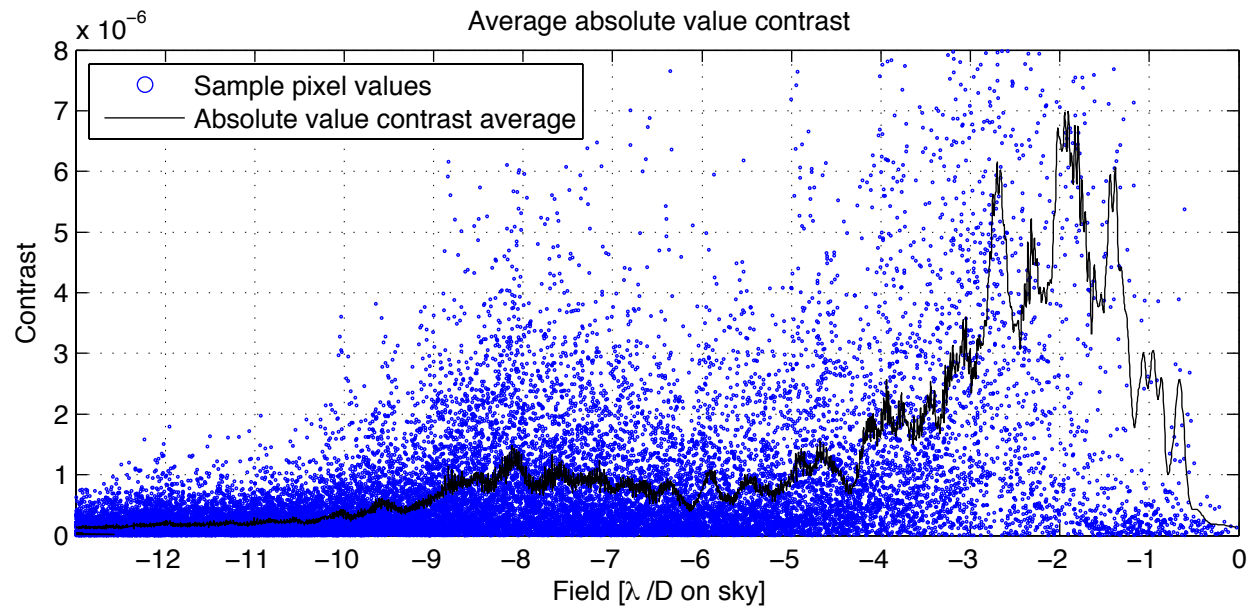

Figure 9. The black line represents the radial absolute value average contrast. The scattered blue dots are the pixel values.

We also computed the radial absolute value average contrast by integrating rings around the central star, thus taking all pixels into account in the calculation. The result is shown as the black line in the Figure 9. The blue dots represent the absolute contrast value of each pixel before averaging them. By using this criteria as a reference to assess 
the diffractive contamination detection limit, we identify the following regions: beyond $12 \lambda / \mathrm{D}$ the contrast envelope is $1.5 \times 10^{-7}$, then it slowly increases to $1 \times 10^{-6}$ at $8 \lambda / \mathrm{D}$, and for smaller field angles it increases to reach a maximum of $5 \times 10^{-6}$ at $2 \lambda / D$. These values are consistent with the results determined by inspection along a cut of the image.

Despite the radial average is a useful criterion to validate the diffraction contamination detection limit; it will easily average out any diffraction contamination. Therefore, our results are still based on image inspection and single cuts.

Table 1: Contrast envelope versus angular separation

\begin{tabular}{|c|c|c|c|c|}
\hline Detectability & \multicolumn{4}{|c|}{ Angular separation $[\lambda / D$ on sky] } \\
\hline limit criteria & $2-5 \lambda / D$ & $5-10 \lambda / \mathrm{D}$ & $10-12 \lambda / \mathrm{D}$ & $>12 \lambda / D$ \\
\hline $\begin{array}{l}\text { Contrast } \\
\text { envelope cut }\end{array}$ & $2.5 \times 10^{-6}$ & $2.5 \times 10^{-6}$ & $5.0 \times 10^{-7}$ & $2.0 \times 10^{-7}$ \\
\hline $\begin{array}{l}\text { Contrast } \\
\text { average }\end{array}$ & $5.0 \times 10^{-6}$ & $1.0 \times 10^{-6}$ & $5.0 \times 10^{-7}$ & $1.5 \times 10^{-7}$ \\
\hline
\end{tabular}

A summary of the contrast regions and their contrast limits to detect diffractive contamination is presented in Table 1 . The limiting factors are ring-like features probably caused by coherent amplification of the different wavefront error sources of the optics. Within this limit, we did not find evidence of diffractive contamination with morphological features that can be generated by a hexagonal dot pattern imprinted on the mirror. This argument suggests that there is no contamination of the IWA to deeper contrast levels; however, this cannot be demonstrated until we can enable the wavefront 
control to suppress the ringing effect. We plan to repeat the experiment when this configuration becomes available.

If an eventual diffractive contamination is found, it would be originated from nonperiodicity of the dot pattern, which would add low-spatial frequency component(s) in the image plane. This component would be speckles in the focal plane, and since these speckles would be coherent with the central source, they can effectively be removed by the wavefront control system. In the case of the AMES high contrast test bed, the wavefront control system is effective to mitigate speckles and other wavefront errors down to $10^{-6}$ to $10^{-8}$ contrast level depending on the working angle (Belikov et al. 2011).

Additional experiments to prove that the diffracted light level can be maintained below 1e-9 in the IWA using a diffractive pupil and polychromatic tests are necessary to complete the compatibility validation of high contrast imaging and a diffractive pupil on the same telescope. These experiments will be performed when a testbed capable of reaching such high contrast becomes available.

\section{REFERENCES}

Belikov, R., et al. 2010, Proc. SPIE, 7731, 2D

Belikov et al. 2011, Proc. SPIE, 8151, 02

Bendek, E., et al. 2011, Proc. SPIE, 8151, 0U

Greene, T., et al. 2007, Bulletin AAS, 39, 975

Guyon, O., 2003, A\&A, 404, 379

Guyon, O., et al. 2005, ApJ, 622, 744

Guyon, O., et al. 2010, Proc. SPIE, 7731, 29 
Guyon, O., et al. 2010, Proc. SPIE, 7736, 24

Guyon, O., et al. 2012, ApJ, 200, 11

Guyon, O., et al. 2012, ApJ, Accepted

Lunine J., et al. 2008, Asbio, Vol 8, Issue 5, pp. 875-881

Marois C., et al. 2006, ApJ 647, 612

Seager, S., et al. 2010, [Exoplanets], University of Arizona Press, Tucson, 162

Sivaramakrishnan \& Oppenheimer 2006, ApJ 647, 620

Shao, M., et al. 2010, ApJ, 720, 357 